\title{
Clinical outcomes after pulmonary vein isolation using an automated tagging module in patients with paroxysmal atrial fibrillation
}

\author{
Min Soo Cho, Jun Kim* ${ }^{*}$, Ungjeong Do, Minsoo Kim, Gi-Byoung Nam, Kee-Joon Choi and You-Ho Kim
}

\begin{abstract}
Background: An automated tagging module (VISITAG ${ }^{\mathrm{TM}}$; Biosense Webster, Irvine, CA) allows objective demonstration of energy delivery. However, the effect of VISITAG ${ }^{\mathrm{TM}}$ on clinical outcomes remains unclear. This study evaluated (1) clinical outcome after AF ablation using VISITAG ${ }^{\mathrm{TM}}$ and (2) the prevalence of gaps in the ablation line.

Methods: This retrospective analysis included 157 consecutive patients (mean age, 56.7 years; $73.2 \%$ men) with paroxysmal atrial fibrillation who underwent successful PVI between 2013 and 2016. Outcomes after the index procedure were compared between those using the VISITAG ${ }^{\mathrm{TM}}$ module (VISITAG group, $n=62$ ) and those not using it (control group, $n=95$ ). The primary outcome was recurrence of AF or atrial tachycardia after a blanking period of 3 months.

Results: The VISITAG group showed significantly shorter overall procedure time (172.2 \pm 37.6 min vs. $286.9 \pm 66.7 \mathrm{~min}, P<0.001)$, ablation time (49.8 $\pm 9.7 \mathrm{~min}$ vs. $82.8 \pm 28.2 \mathrm{~min}, P<0.001)$, and fluoroscopy time $(11.8 \pm 5.3 \mathrm{~min}$ vs. $34.2 \pm 30.1 \mathrm{~min}, P<0.001)$ compared with controls. The 1 -year recurrence-free survival rate was not statistically different between the groups (70.8\% in the VISITAG group vs. $79.2 \%$ in the control group, $P=0.189)$. Gaps in the VISITAG line were common in the both carina and left side pulmonary veins. Patients without gaps ( $\geq 5 \mathrm{~mm}$ ) by the criteria emphasizing catheter stability ( $>15 \mathrm{~s},<4 \mathrm{~mm}$ range, $>60 \%$ force over time, $>6 \mathrm{~g}$ contact force) showed higher recurrence-free survival rate compared with those with gaps (borderline statistical significance, $91.7 \%$ vs. $66.0 \%, P=0.094)$.
\end{abstract}

Conclusion: Use of the VISITAG ${ }^{\mathrm{TM}}$ module significantly reduced procedure, ablation, and fluoroscopic times with a similar AF/AT recurrence rate compared with the conventional ablation. Clinical implications of minimizing gaps along the ablation line should be evaluated further in the future prospective studies.

Keywords: Atrial fibrillation, Catheter ablation, Radiofrequency, Recurrence

\section{Introduction}

The radiofrequency catheter ablation (RFCA) of atrial fibrillation (AF) has evolved in recent years to become first or secondary line of treatment option for rhythm control [1-3]. It is more effective in terms of sinus rhythm maintenance and minimizing the long-term

${ }^{*}$ Correspondence: mdjunkim@gmail.com

Department of Cardiology, Asan Medical Center, University of Ulsan College of Medicine, 88, Olympic-ro 43-gil, Songpa-gu, Seoul 05505, South Korea adverse events associated with anti-arrhythmic drugs [4]. CARTO VISITAG ${ }^{\mathrm{TM}}$ (Biosense Webster, Irvine, CA) annotates the ablation site objectively whenever predefined criteria such as catheter stability, time, contact force, or impedance drop are fulfilled.

If there were gaps in the tagging by $\operatorname{VISITAG}^{\mathrm{TM}}$, it might reflect non-contiguous lesion delivery which could explain pulmonary vein reconnection [5]. However, the optimal criteria of gap have not yet been settled to date. Lin et al. [6] and Okumura et al. [7] recently suggested criteria for the VISITAG gap based on their experience

c) The Author(s) 2020. This article is licensed under a Creative Commons Attribution 4.0 International License, which permits use, sharing, adaptation, distribution and reproduction in any medium or format, as long as you give appropriate credit to the original author(s) and the source, provide a link to the Creative Commons licence, and indicate if changes were made. The images or other third party material in this article are included in the article's Creative Commons licence, unless indicated otherwise in a credit line to the material. If material is not included in the article's Creative Commons licence and your intended use is not permitted by statutory regulation or exceeds the permitted use, you will need to obtain permission directly from the copyright holder. To view a copy of this licence, visit http://creativeco mmons.org/licenses/by/4.0/. 
and data on acute recurrence. However, as these studies were based on experts' opinions rather than long-term clinical outcomes, the implications of VISITAG gap are still not well evaluated. In the current study, we hypothesized that the gaps in the VISITAG ${ }^{\mathrm{TM}}$ are associated with long-term clinical outcomes and tested them in patients with paroxysmal AF who underwent first pulmonary vein isolation (PVI).

\section{Methods}

\section{Study population}

Of the 451 patients who underwent RFCA for AF using the CARTO (Biosense Webster, Irvine, CA) threedimensional mapping system between November 2013 and March 2016 at Asan Medical Center in Seoul, South Korea, we included PAF patients who underwent first PV isolation. We excluded patients who had any of the following: (1) persistent AF $(n=173)$; (2) previous RFCA $(n=37)$; or (3) previous maze operation $(n=2)$, additional left atrial line or complex fractionated atrial electrogram ablation $(n=63)$, incomplete PV isolation $(n=4)$, and incomplete data $(n=15)$. The main study population was comprised of 157 patients with or without cavotricuspid isthmus ablations (Fig. 1). The patients were divided into two groups according to the use of the VISITAG $^{\mathrm{TM}}$ module during the procedure (with
VISITAG, $n=62$; without VISITAG, $n=95$ ). This study conformed to the ethical guidelines of the 1975 Declaration of Helsinki and was approved by the Institutional Review Board of Asan Medical Center (2017-0357). The need for informed consent was waived due to the retrospective nature of the study.

\section{Ablation procedure and follow-up}

The demographic, electrocardiographic, and echocardiographic characteristics of the patients were obtained by independent research personnel at the time of admission for the RFCA procedure. A duodecapolar catheter was initially placed in the coronary sinus and right atrium. Double transseptal catheterization was performed under hemodynamic monitoring and fluoroscopic guidance, one for mapping and one for the ablation catheter. A 7 Fr Lasso catheter or Pentaray catheter (Biosense Webster, Irvine, CA) was placed for mapping. Analgesia was maintained with remifentanil throughout the procedure. Electroanatomical mapping and ablation procedures were performed using the $\mathrm{CARTO}^{\circledR}$ system (Biosense Webster, Irvine, CA). Mapping and ablation were completed using a 7.5 Fr NaviStar SmartTouch SF irrigation catheter with a $3.5 \mathrm{~mm}$ tip (Biosense Webster). We performed PVI using a target contact force of 10-30 grams and delivered energy of 30-35 watts

\section{A total of 451 patients who underwent AF-RFCA using} CARTO system (Jan. 2013- Dec. 2016)

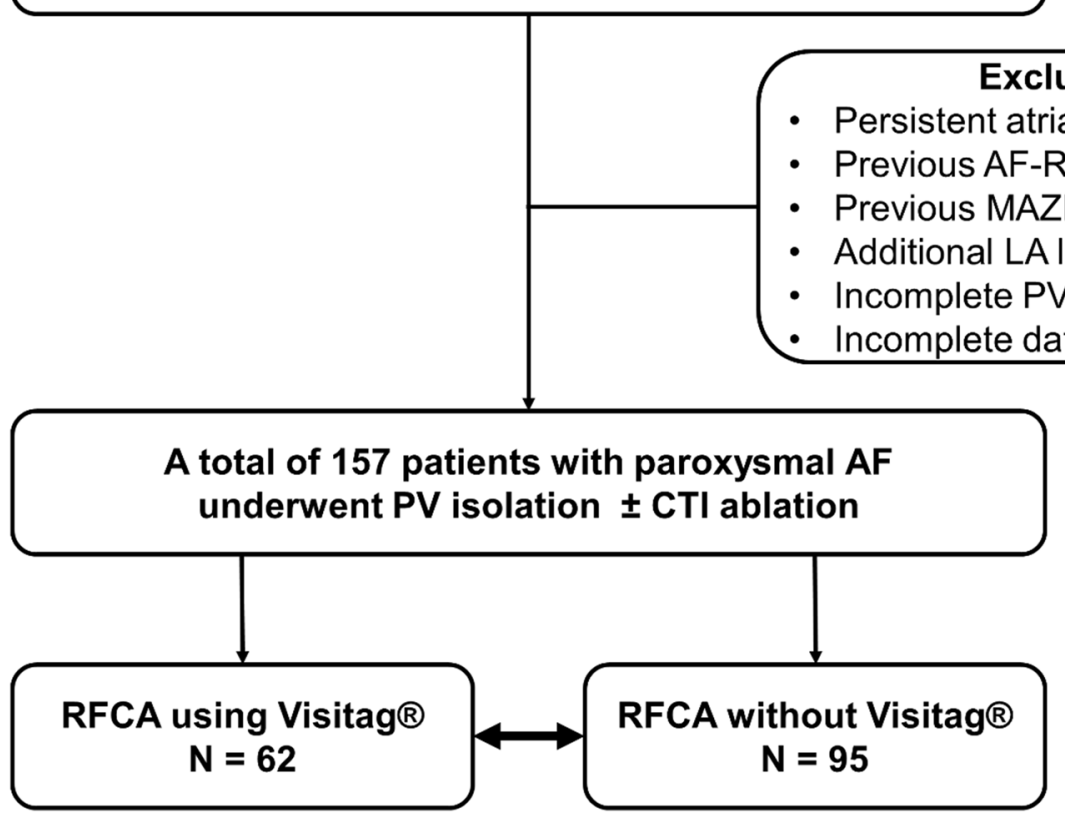

Fig. 1 Study flow diagram 
(power control mode) for the anterior wall and 25-30 watts for the posterior wall. In the VISITAG group, the ablation lesions were tagged automatically based on predefined criteria on the VISITAG module (Fig. 2). Ablation lines were made sequential point-by-point fashion to avoid gaps in the line with VISITAG ${ }^{\mathrm{TM}}$ setting of our center (minimum time of $5 \mathrm{~s}$, maximum range of $2.5 \mathrm{~mm}$, force over time of $50 \%$, minimum force of $5 \mathrm{~g}$, and non-projected tags). Manual or projected tags were not used in VISITAG group. In the control group, we delivered a point-by-point RF application in a powercontrolled mode with same energy setting as VISITAG group. We tagged the ablation lesions manually according to the physician's discretion, based on the reduction in bipolar electrogram during ablation as well as drop of the local impedance.

The procedural endpoint was the complete entrance block of all $4 \mathrm{PVs}$, defined as elimination of all ostial PV potentials during sinus or paced rhythm in both groups. Additional ablation between gaps along the intended ablation line was not done after the electrical isolation of the PV from the left atrium. A 30-minute waiting period was observed to monitor for early reconnection. Uninterrupted anticoagulation, either using warfarin or non-vitamin $\mathrm{K}$ antagonist oral anticoagulants, was maintained before and after the procedure. Activated clotting time was adjusted for $300-350 \mathrm{~s}$ during the procedure. Patient follow-up was conducted at $1,3,6$, and 12 months after the index procedure with 12-lead electrocardiogram and 24-hour Holter monitoring. Additional rhythm monitoring was performed if the patient had symptoms suggestive of arrhythmic recurrence.

\section{Gap analysis}

The gaps in the ablation line were assessed according to three predefined criteria for VISITAG ${ }^{\mathrm{TM}}$ tagging. Criterion 1 followed the VISITAG ${ }^{\mathrm{TM}}$ settings of our center which described above (minimum time of $5 \mathrm{~s}$, maximum range of $2.5 \mathrm{~mm}$, force over time of $50 \%$, minimum force of $5 \mathrm{~g}$ ). For Criterion 2, the tagging was repeatedly assessed using the criteria established by Lin et al. [6], which consisted of a minimum time of $15 \mathrm{~s}$, a maximum range of $4 \mathrm{~mm}, 60 \%$ force over time, and $6 \mathrm{~g}$ minimum force. For Criterion 3, we reassessed the gaps by applying the criteria reported by Okumura et al. [7], which consisted of a minimum time of $5 \mathrm{~s}$, a maximum range of $3 \mathrm{~mm}, 25 \%$ force over time, and $8 \mathrm{~g}$ minimum force. Gaps in the VISITAG line were defined as a margin-tomargin distance of $5 \mathrm{~mm}$ between two adjacent VISITAG $^{\mathrm{TM}}$ tags (Fig. 2). The gaps in the line were assessed by two independent research personnel who were blinded to the study outcomes. The electroanatomical maps were rotated three-dimensionally to avoid inadequate measurement by angle. Interobserver variability was assessed by gap analysis of 20 randomly selected samples and demonstrated excellent consistency (kappa value, 0.88). Conflicted cases were resolved by the consensus.

\section{Study outcome}

The primary study outcome was rate of recurrence of AF or atrial tachycardia (AT) after a blanking period of 3 months. The recurrence of AF or AT was diagnosed when a sustained episode lasting $>30 \mathrm{~s}$ was documented on the electrocardiogram or Holter monitoring, either routine or symptom driven. The secondary outcome was the location and prevalence of VISITAG gap by each of

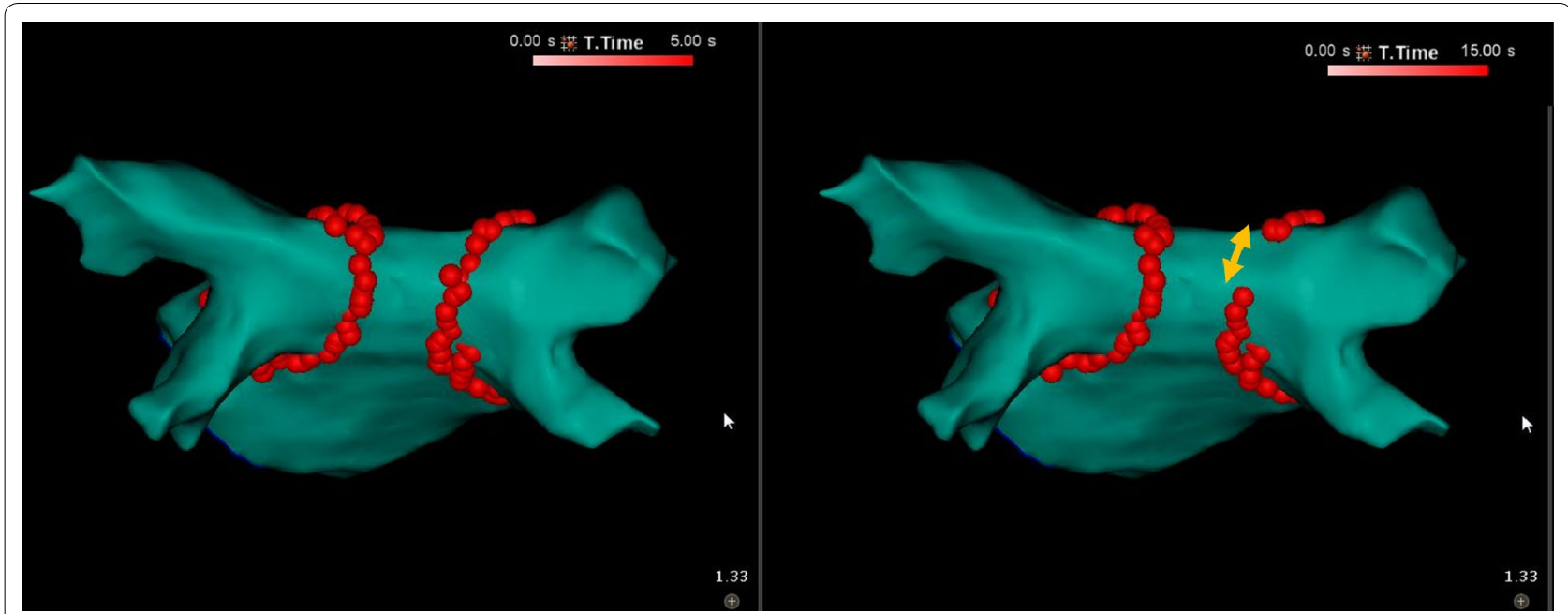

Fig. 2 Representative case of gap analysis. There were no gaps when using Criterion 1 (left), but gaps were noted when Criterion 2 was applied (right) 
the three criteria. In addition, the rate of recurrent $\mathrm{AF}$ and AT was reassessed according to the presence or absence of VISITAG gap on the PVI line to assess the clinical impact of such gaps. Total procedure time, ablation time, fluoroscopic time, and procedure-related complications after PVI were also evaluated in the current study. All clinical outcomes were verified and adjudicated by an independent researcher.

\section{Statistical analysis}

All statistical analyses were performed using R software (version 3.3.1; R Foundation for Statistical Computing, Vienna, Austria; www.r-project.org). All $P$ values were two-sided, and $P$ values $<0.05$ were considered statistically significant. Continuous variables were presented as either means with standard deviation or median values with interquartile ranges. Categorical variables were presented as frequency and percentage. Continuous variables were compared using Student's $t$ test or the Wilcoxon rank-sum test. Categorical variables were compared using the $\chi^{2}$ test or Fisher's exact test, as appropriate. The cumulative rates of recurrent AF and AT were demonstrated using Kaplan-Meyer analysis with differences assessed using the Log-rank test. Cox's proportional hazard model was used to demonstrate the association between gap and recurrence of AF and AT during follow-up.

\section{Results}

Between March 2009 and December 2016, a total of 451 patients underwent AF-RFCA using the CARTO system in our center. Among these patients, 157 paroxysmal AF patients underwent PVI with or without use of VISITAG $^{\mathrm{TM}}$. In the baseline characteristics (Table 1), the mean age of the patients was $56.7 \pm 10.9$ years, $73.2 \%$ were men, and the mean $\mathrm{CHA}_{2} \mathrm{DS}_{2}$-VASc score was $1.2 \pm 1.4$ points. There were no significant differences in baseline characteristics between those with and without VISITAG $^{\mathrm{TM}}$ use. The left atrial (LA) anterior-posterior diameter ( $39.4 \pm 4.9$ vs. $39.9 \pm 6.3 \mathrm{~mm}, p=0.554)$ or LA volume of the CARTO three-dimensional electroanatomical map (116.6 \pm 31.8 vs. $108.0 \pm 33.8 \mathrm{ml}, p=0.147)$ was not significantly different between the two groups.

Procedural characteristics are summarized in Table 2. A contact force sensing catheter was mostly used in those patients for whom VISITAG ${ }^{\mathrm{TM}}$ was used. The rate of cavotricuspid isthmus ablation in addition to the PVI was not different between two groups. The procedure time $(286.9 \pm 66.7 \mathrm{~min}$ vs. $172.2 \pm 37.6 \mathrm{~min} ; P<0.001)$ and ablation time $(82.8 \pm 28.2 \mathrm{~min}$ vs. $49.8 \pm 9.7 \mathrm{~min}$; $P<0.001)$ were significantly shorter in VISITAG group.

Table 1 Baseline characteristics of the study patients

\begin{tabular}{|c|c|c|c|c|}
\hline & $\begin{array}{l}\text { Overall } \\
(N=157)\end{array}$ & $\begin{array}{l}\text { VISITAG }(-) \\
(N=95)\end{array}$ & $\begin{array}{l}\text { VISITAG }(+) \\
(N=62)\end{array}$ & $P$ value \\
\hline Age & $56.7 \pm 10.9$ & $55.6 \pm 11.2$ & $58.5 \pm 10.2$ & 0.099 \\
\hline Men & $115(73.2)$ & $70(73.7)$ & $45(72.6)$ & $>0.99$ \\
\hline BMI & $24.6 \pm 3.4$ & $24.8 \pm 3.1$ & $24.3 \pm 3.8$ & 0.339 \\
\hline \multicolumn{5}{|l|}{ Previous medical history } \\
\hline Hypertension & $51(32.5)$ & $32(33.7)$ & $19(30.6)$ & 0.823 \\
\hline Diabetes & $14(8.9)$ & $8(8.4)$ & $6(9.7)$ & $>0.99$ \\
\hline Congestive heart failure & $3(1.9)$ & $2(2.1)$ & $1(1.6)$ & $>0.99$ \\
\hline Vascular disease & $4(2.5)$ & $3(3.2)$ & $1(1.6)$ & 0.934 \\
\hline Ischemic stroke/TIA & $16(10.2)$ & $6(6.3)$ & $10(16.1)$ & 0.086 \\
\hline $\mathrm{CHA}_{2} \mathrm{DS}_{2}$-VASc score & $1.2 \pm 1.4$ & $1.1 \pm 1.4$ & $1.4 \pm 1.3$ & 0.284 \\
\hline $\mathrm{CHA}_{2} \mathrm{DS}_{2}$-VASc score $\geq 2$ & $54(34.4)$ & $28(29.5)$ & $26(41.9)$ & 0.151 \\
\hline Left ventricular ejection fraction, $\%$ & $61.0 \pm 6.3$ & $61.0 \pm 6.1$ & $60.9 \pm 6.7$ & 0.905 \\
\hline Left atrial size, $\mathrm{mm}$ & $39.6 \pm 5.5$ & $39.4 \pm 4.9$ & $39.9 \pm 6.3$ & 0.554 \\
\hline Left atrial volume, $\mathrm{ml}$ & $112.5 \pm 32.9$ & $116.6 \pm 31.8$ & $108.0 \pm 33.8$ & 0.147 \\
\hline Amiodarone & $14(8.9)$ & $8(8.4)$ & $6(9.7)$ & $>0.99$ \\
\hline Class 1C drug & $120(76.4)$ & $68(71.6)$ & $52(83.9)$ & 0.114 \\
\hline Beta-blocker & $47(29.9)$ & $22(23.2)$ & $25(40.3)$ & 0.034 \\
\hline Calcium channel blockers & $47(29.9)$ & $32(33.7)$ & $15(24.2)$ & 0.275 \\
\hline Digoxin & $10(6.4)$ & $2(2.1)$ & $8(12.9)$ & 0.018 \\
\hline
\end{tabular}

$B M I$ body mass index; TIA transient ischemic attack

Data are presented as mean \pm standard deviation or number (\%) 
Table 2 Procedural characteristics

\begin{tabular}{|c|c|c|c|c|}
\hline & $\begin{array}{l}\text { Overall } \\
(N=157)\end{array}$ & $\begin{array}{l}\text { VISITAG }(-) \\
(N=95)\end{array}$ & $\begin{array}{l}\text { VISITAG }(+) \\
(N=62)\end{array}$ & $P$ value \\
\hline Contact force catheter & $74(47.1)$ & $12(12.6)$ & $62(100.0)$ & $<0.001$ \\
\hline Cavotricuspid isthmus ablation & $97(61.8)$ & $63(66.3)$ & $34(54.8)$ & 0.201 \\
\hline Procedure time (min) & $241.6 \pm 80.0$ & $286.9 \pm 66.7$ & $172.2 \pm 37.6$ & $<0.001$ \\
\hline Ablation time (s) & $69.8 \pm 27.9$ & $82.8 \pm 28.2$ & $49.8 \pm 9.7$ & $<0.001$ \\
\hline Fluoroscopy time (min) & $25.3 \pm 26.0$ & $34.2 \pm 30.1$ & $11.8 \pm 5.3$ & $<0.001$ \\
\hline Procedure-related complications & $0(0)$ & $(0)$ & $0(0)$ & \\
\hline
\end{tabular}

Data are presented as mean \pm standard deviation or number (\%)

The fluoroscopic time was also significantly shorter in this group $(34.2 \pm 30.1 \mathrm{~min}$ vs. $11.8 \pm 5.3 \mathrm{~min} ; P<0.001)$. There were no major procedure-related complications in either group.

The prevalence of the gaps determined by each criterion is depicted in Fig. 3. According to Criterion 1, gaps in the ablation line were noted in $38.7 \%$ of patients (Fig. 3a) and were prevalent in both carina segments. When Criterion 2 was applied, the prevalence of gaps increased significantly $(80.6 \%, P<0.001$ vs. Criterion 1; Fig. 3b), especially those at the right roof $(24.2 \%$ vs. $11.3 \%, P=0.060)$ and left ridge (22.6\% vs. $8.1 \%, P=0.025)$ compared with Criterion 1. When applying Criterion 3, the overall prevalence of gaps was not significantly different (50\%, $P=0.206$ vs. Criterion 1 ; Fig. 3c). In this setting, the lower ridge segment showed a numerically higher rate of gap compared with the default criteria $(21.0 \%$ vs. 9.7\%, $P=0.081$ ).

There were 37 cases of recurrence (23.5\%) during 1 year after the blanking period. The cumulative recurrent AFand AT-free survival rate was not significantly different between the groups with and without VISITAG (79.2\% vs. $70.8 \%, P=0.189$; Fig. 4 a). The patients without VISITAG gap showed a numerically higher recurrence-free survival rate (Fig. 4b-d), but without statistical significance compared with Criterion 1 (Fig. 4b) and Criterion 3 (Fig. 4d). When Criterion 2 was applied, the presence of VISITAG gap was associated with a lower recurrencefree survival rate compared with those without gaps (borderline statistical significance, $91.7 \%$ vs. $66.0 \%, P=0.094$;

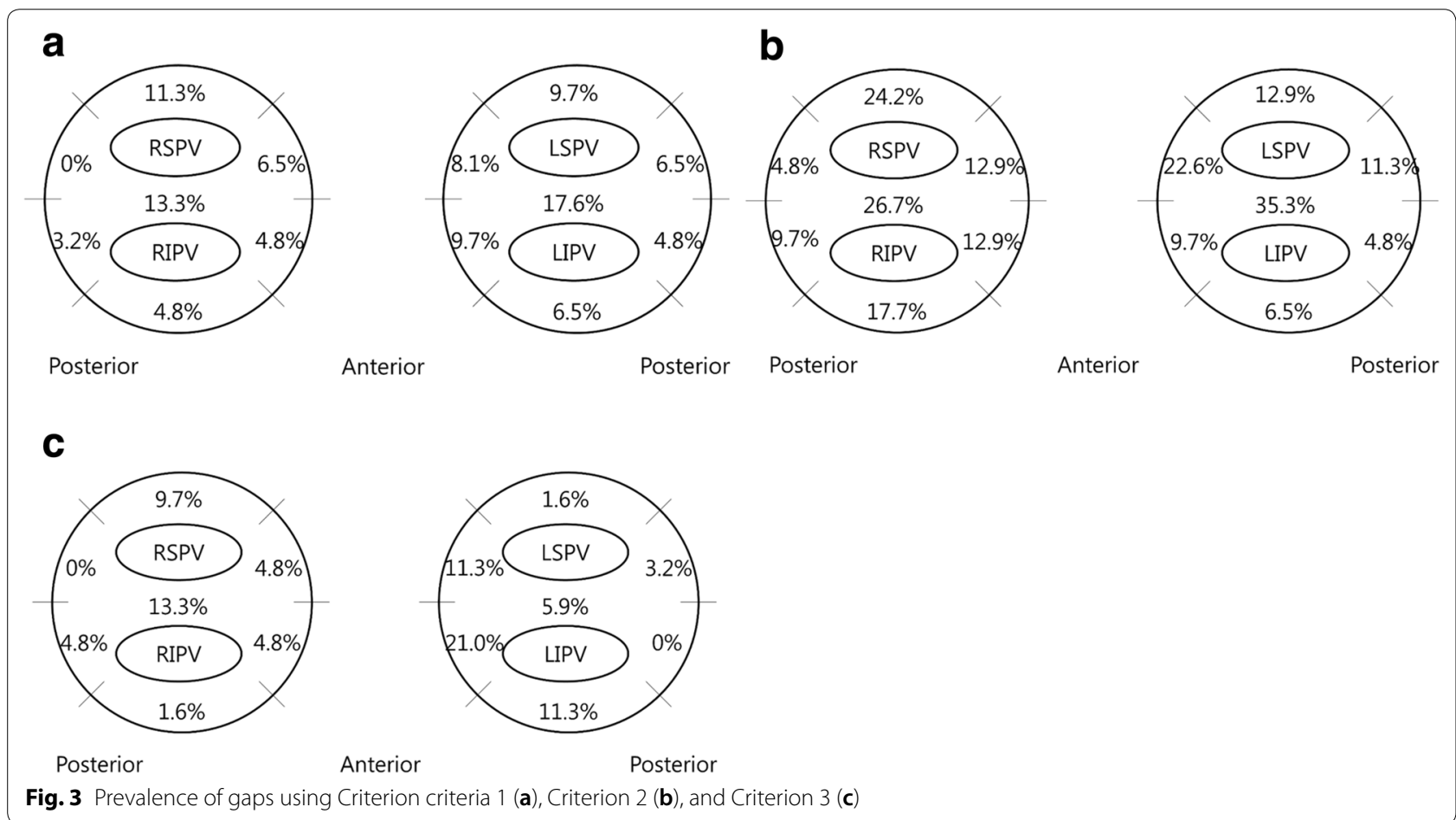




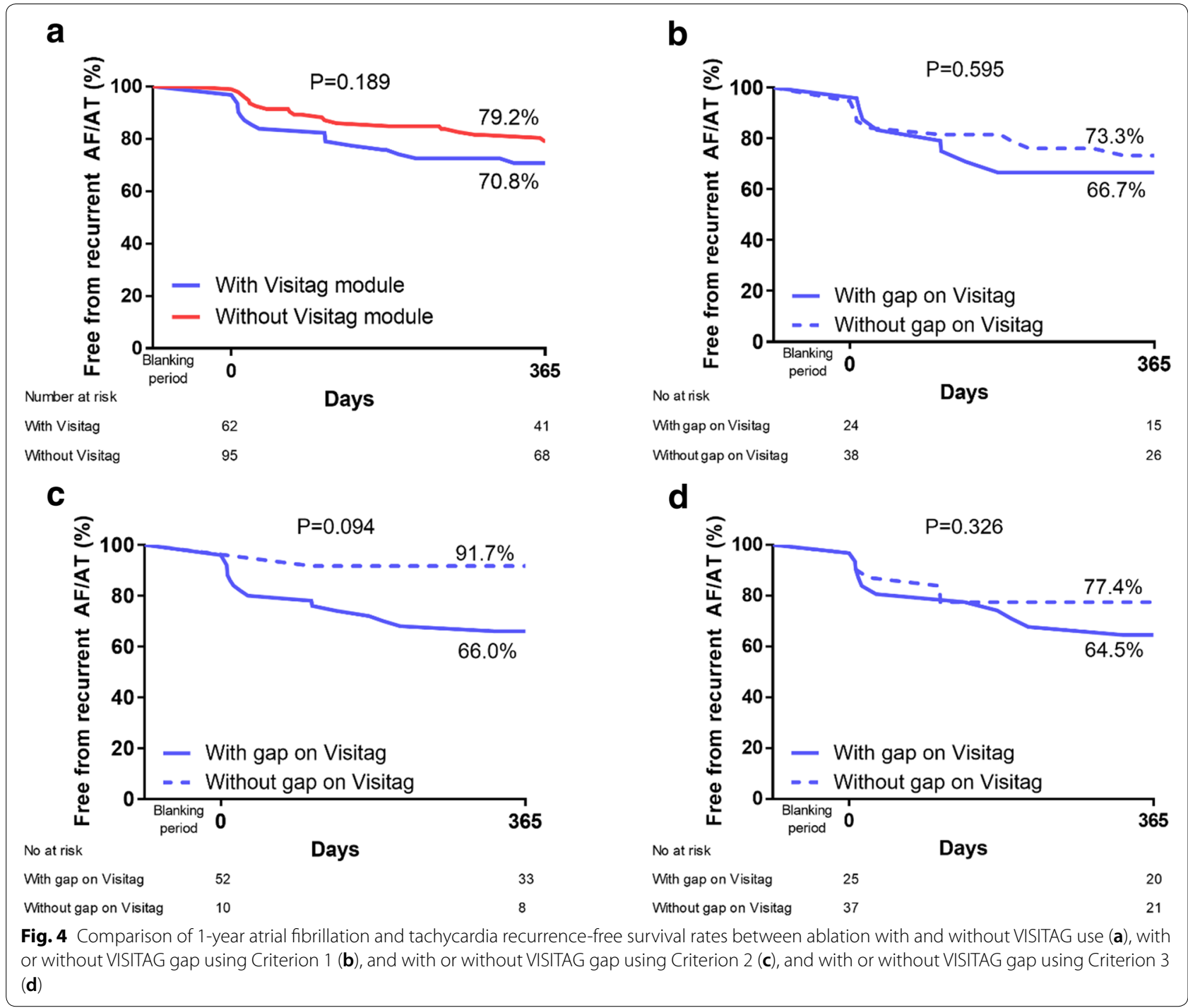

Fig. 4c). The sensitivity, specificity, positive predictive value, and negative predictive value of the VISITAG gap by Criterion 2 to the AF and AT recurrence were 94.7\%, $25.6 \%, 36.0 \%$ and $91.7 \%$, respectively.

\section{Discussion}

In this retrospective study, we report several clinically relevant findings regarding the implication of VISITAG ${ }^{\text {TM }}$ on the AF-RFCA. The use of VISITAG ${ }^{\mathrm{TM}}$ shortens the procedure time, ablation time, and fluoroscopic time without significant difference in AF and AT recurrence. Gaps in the line were most prevalent in both carina in Criterion 1, the right roof and left upper ridge by Criterion 2 , and the left lower ridge by Criterion 3 . The presence of gaps by the Criterion 2 was associated with lower recurrence-free survival rate compared with those without VISITAG use.
There have been several efforts made for durable, homogenous transmural lesions. The clinical usefulness of VISITAG ${ }^{\mathrm{TM}}$ is partly assessed in the previous study [8], in which the VISITAG module is associated with a higher first-pass PV isolation with a shorter ablation time. The recently published CLOSE protocol made extreme efforts regarding delivery of constant energy and force with sufficient duration in contiguous lesions, and their excellent efficacy has already been proven in both animal and clinical studies [8-10]. The VISITAG ${ }^{\mathrm{TM}}$ is a fundamental component of such point-by-point approaches, as it objectively demonstrates the gaps in the line that are inadequately ablated regions associated with future reconnections [11]. Therefore, the analysis of the gaps in our study is clinically important, as it could reveal the missing points in our practice and ultimately enhance outcomes after the procedure. 
In our study, use of VISITAG was associated with similar AF- and AT-free survival rates, despite a $40 \%$ reduction in ablation times. In addition, use of this module resulted in a $40 \%$ reduction in the overall procedure time and a $66 \%$ reduction in fluoroscopic time. We believe this is a clinically important finding, as shorter procedure, ablation, and radiation times are critical factors in terms of patient safety. Longer procedure and ablation times are possibly associated with more procedure-related complications and radiation exposure [12]. With objective tagging of criteria-fulfilled lesions, operators can avoid unnecessary burns. On the contrary, gaps in the line intuitively indicate regions to be ablated further, which might explain the reduced ablation time with similar efficacy in our data [6]. We therefore believe the VISITAG module is a clinically useful tool for safe and effective ablation.

In the current study, distribution and implication of gaps in the VISITAG ${ }^{\mathrm{TM}}$ module varied according to the criterion applied. Using the default setting, gaps were prevalent in the carina, which reflects difficulties in maintaining catheter stability in this region. Prevalence of gaps was exaggerated in the RSPV roof and left upper ridge area using Criterion 2. As Criterion 2 focused on extremely long stability ( $>15 \mathrm{~s}$ ), this finding reflects the difficulties of catheter positioning in this area. It also demonstrates that the stability of catheter is important as gaps by Criterion 2 are associated with a lower arrhythmia-free survival rate. Recently published data from Reddy et al. stressed that catheter stability is more important than force itself and that it is associated with better long-term outcomes [13]. In their study, which is in line with ours, difficulties in maintaining stability in both carina and left PVs were noted. This means that special care should be taken during ablation of these regions. Application of looping techniques could be a complementary method to ensure stability [14]. Criterion 3 focused on higher force $(>8 \mathrm{~g}$ ) than the default setting, and therefore, gaps demonstrated a different distribution prevalent in the lower ridge area, reflecting the difficulties maintaining high force in this area [7]. Use of a deflectable sheath or sheath mediated torque would be helpful [15]. The absence or presence of gaps by the Criterion 3 did not affect the long-term outcomes. The differential outcome data of Criterion 2 and 3 were partially explained by the recent data from Chubb et al., which compared VISITAG gap to the atrial scar in the post-procedure cardiac magnetic resonance imaging (MRI) [16]. The mature atrial scar increased linear fashion with increased ablation time criteria, but not with the stringent force criteria (>10 g): The scar was frequently created at lower force. Therefore, we believe contiguous atrial scar formation, which reflected in absence of gaps by Criterion 2 emphasizing longer ablation time, might lead to better clinical outcomes. Our results are in line with aforementioned study by Reddy et al. [13].

\section{Limitations}

There were several limitations in this study. The retrospective observational design of the study entails inherent selection bias. Because the result is from the single-center registry, the procedure pattern is specific to our center. Results could be different if the same analysis is performed in another center. Because the VISITAG group enrolled later than the control group, the difference in procedure characteristics (such as ablation or procedure times) is partly affected by the changing practice or increasing experience of the operator rather than VISITAG use. The number of patients in the VISITAG analysis is too small to derive a statistically significant difference. Therefore, we cannot determine the optimal VISITAG settings, such as number, length, or locations based on the current data. For the same reason, generalization of our findings should be made with caution. Further evaluation with larger prospective cohorts, including comparison with objective "gold standard" such as cardiac MRI or reconnections in the repeated procedure, is needed.

\section{Conclusion}

Use of the VISITAG ${ }^{\mathrm{TM}}$ module significantly reduced procedure, ablation, and fluoroscopic times with similar AT and AF recurrence rates compared with conventional ablation methods. The clinical implications of gaps in the ablation line should be evaluated further in future prospective studies.

\section{Abbreviations \\ AF: Atrial fibrillation; AT: Atrial tachycardia; CTI: Cavotricuspid isthmus; LA: Left atrium; MRI: Magnetic resonance imaging; PV: Pulmonary vein; RFCA: Radi- ofrequency catheter ablation.}

\section{Acknowledgements}

We are grateful to Arasil Lee and Hyang-Gi Son for their excellent support in image processing and analysis. We did not receive any specific grants from funding agencies in the public, commercial, or not-for-profit sectors for this research.

\section{Authors' Contributions}

$\mathrm{MC}$ analyzed and interpreted the patient data and a major contributor in writing the manuscript. UD and MK analyzed the patient data. GN, KC, and YK supervised the interpretation of the data and the manuscript. JK analyzed and made a final decision on the manuscript. All authors read and approved the final manuscript.

\section{Funding}

Not applicable.

\section{Availability of data and materials}

The datasets used and/or analyzed during the current study are available from the corresponding author on reasonable request. 


\section{Compliance with ethical standards}

\section{Ethics approval and consent to participate}

This study was approved by the Institutional Review Board of Asan Medica Center (Seoul, 64 South Korea; Institutional Review Board No. 2017-0357).

\section{Consent for publication}

Not applicable.

\section{Competing interests}

The authors declare that they have no competing interests.

Received: 29 May 2020 Accepted: 31 July 2020

Published online: 08 September 2020

\section{References}

1. Calkins H, Kuck KH, Cappato R, Brugada J, Camm AJ, Chen SA, et al. 2012 HRS/EHRA/ECAS Expert Consensus Statement on Catheter and Surgical Ablation of Atrial Fibrillation: recommendations for patient selection, procedural techniques, patient management and follow-up, definitions, endpoints, and research trial design. Europace: Eur Pacing Arrhythm Cardiac Electrophysiol J Working Groups Cardic Pacing Arrhythm Cardiac Cell Electrophysiol Eur Soc Cardiol. 2012;14:528-606.

2. Kirchhof P, Benussi S, Kotecha D, Ahlsson A, Atar D, Casadei B, et al. 2016 ESC Guidelines for the management of atrial fibrillation developed in collaboration with EACTS. Eur Heart J. 2016;37:2893-962.

3. Park H-S, Jeong DS, Yu HT, Pak H-N, Shim J, Kim JY, et al. Korean guidelines for catheter ablation of atrial fibrillation: Part I. Int J Arrhythm. 2018;19(3):186-234

4. Khan AR, Khan S, Sheikh MA, Khuder S, Grubb B, Moukarbel GV. Catheter ablation and antiarrhythmic drug therapy as first- or second-line therapy in the management of atrial fibrillation: systematic review and metaanalysis. Circul Arrhythm Electrophysiol. 2014;7:853-60.

5. Ouyang F, Antz M, Ernst S, Hachiya H, Mavrakis H, Deger FT, et al. Recovered pulmonary vein conduction as a dominant factor for recurrent atrial tachyarrhythmias after complete circular isolation of the pulmonary veins: lessons from double Lasso technique. Circulation. 2005;111:127-35

6. Lin T, Ouyang F, Kuck K-H, Tilz R. THERMOCOOL ${ }^{\circledR}$ SMARTTOUCH $^{\circledR} \mathrm{CATH}-$ ETER-The evidence so far for contact force technology and the role of VISITAG ${ }^{\text {TM }}$ MODULE. Arrhythm Electrophysiol Rev. 2014;3:44

7. Okumura Y, Watanabe I, Iso K, Nagashima K, Sonoda K, Sasaki N, et al. Clinical utility of automated ablation lesion tagging based on catheter stability information (VisiTag Module of the CARTO 3 System) with contact force-time integral during pulmonary vein isolation for atrial fibrillation. J Interv Cardiac Electrophysiol Int J Arrhythm Pacing. 2016;47:245-52.

8. Phlips T, Taghji P, El Haddad M, Wolf M, Knecht S, Vandekerckhove Y, et al. Improving procedural and one-year outcome after contact force-guided pulmonary vein isolation: the role of interlesion distance, ablation index, and contact force variability in the 'CLOSE'-protocol. EP Europace. 2018:20:f419-27.

9. Duytschaever M, De Pooter J, Demolder A, El Haddad M, Phlips T, Strisciuglio T, et al. Long-term impact of catheter ablation on arrhythmia burden in low-risk patients with paroxysmal atrial fibrillation: the CLOSE to CURE study. Heart Rhythm. 2020;17(4):535-43.

10. Berte B, Hilfiker G, Moccetti F, Schefer T, Weberndörfer V, Cuculi F, et al. Pulmonary vein isolation using ablation index vs. CLOSE protocol with a surround flow ablation catheter. Europace. 2020;22(1):84-9.

11. De Pooter J, Strisciuglio T, El Haddad M, Wolf M, Phlips T, Vandekerckhove $Y$, et al. Pulmonary vein reconnection no longer occurs in the majority of patients after a single pulmonary vein isolation procedure. JACC: Clin Electrophysiol. 2019:5:295-305

12. Gupta A, Perera T, Ganesan A, Sullivan T, Lau DH, Roberts-Thomson KC, et al. Complications of catheter ablation of atrial fibrillation: a systematic review. Circul Arrhythm Electrophysiol. 2013;6:1082-8.

13. Reddy $\mathrm{VY}$, Pollak S, Lindsay BD, McElderry HT, Natale A, Kantipudi C, et al. Relationship between catheter stability and 12-month success after pulmonary vein isolation: a subanalysis of the SMART-AF trial. JACC Clin Electrophysiol. 2016;2:691-9.

14. Kyriakopoulou M, Strisciuglio T, El Haddad M, De Pooter J, Almorad A, Van Beeumen $\mathrm{K}$, et al. Evaluation of a simple technique aiming at optimizing point-by-point isolation of the left pulmonary veins: a randomized study. Europace Eur Pacing Arrhythm Cardiac Electrophysiol J Work Groups Cardiac Pacing Arrhythm Cardiac Cell Electrophysiol Eur Soc Cardiol. 2019:21:1185-92.

15. Kimura T, Takatsuki S, Oishi A, Negishi M, Kashimura S, Katsumata Y, et al. Operator-blinded contact force monitoring during pulmonary vein isolation using conventional and steerable sheaths. Int J Cardiol. 2014;177:970-6.

16. Chubb H, Lal K, Kiedrowicz R, Karim R, Williams SE, Harrison J, et al. The value of ablation parameter indices for predicting mature atrial scar formation in humans: an in vivo assessment using cardiac magnetic resonance imaging. J Cardiovasc Electrophysiol. 2019;30:67-77.

\section{Publisher's Note}

Springer Nature remains neutral with regard to jurisdictional claims in published maps and institutional affiliations.
Ready to submit your research? Choose BMC and benefit from:

- fast, convenient online submission

- thorough peer review by experienced researchers in your field

- rapid publication on acceptance

- support for research data, including large and complex data types

- gold Open Access which fosters wider collaboration and increased citations

- maximum visibility for your research: over 100M website views per year

At BMC, research is always in progress.

Learn more biomedcentral.com/submissions 